\title{
DIFFERENTIAL DIAGNOSIS OF HIP CONTRACTURES IN PARAPLEGICS AND THEIR TREATMENT
}

\author{
By R. GassLer, M.D. \\ Rehabilitationszentrum der Allgemeinen Unfallversicherungsanstalt \\ Tobelbad bei Graz. Austria
}

SPASMS are inevitable consequences of central paralysis. From experience it is known that very severe spasms cannot be improved significantly by conservative treatment, or only for a short period. The intensity of myospasms does not decrease even after many months or years, and therefore, considerable contractures may develop. However, contractures can develop in a very short time, i.e. a few weeks.

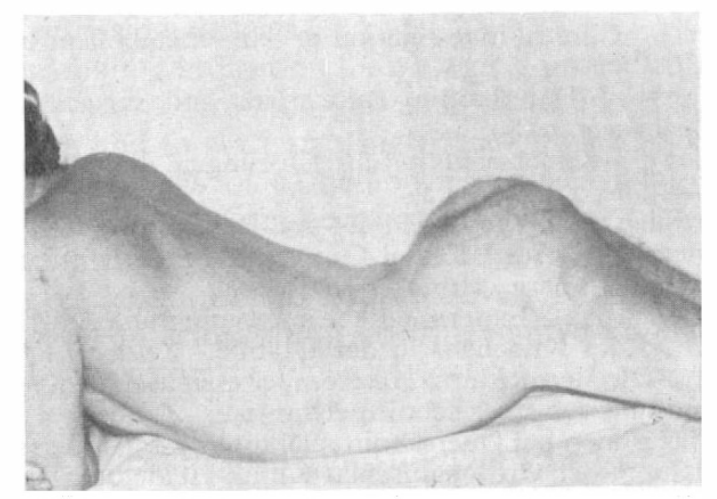

FIG. I

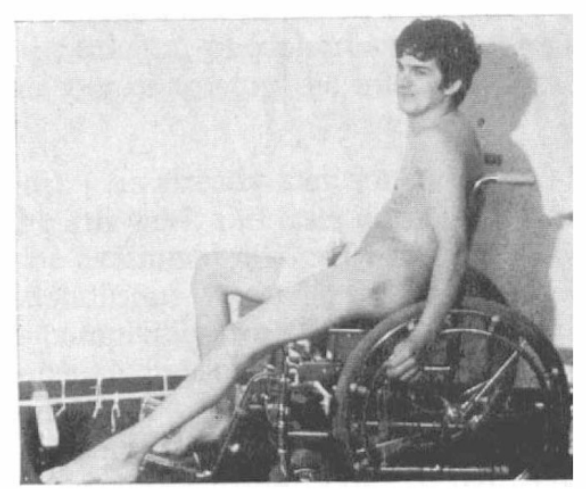

FIG. 2

In paraplegics, we have to deal mainly with contractures due to spasticity of the hip and knee joints. The spasms of muscle groups which are clinically pre- 
ponderant lead to typical malposition of the joints such as adduction and flexion contractures of the hip; flexion contractures of the knee joint and talipes equinus due to the overaction of the calf muscles. Extension contractures of the hip and knee do not occur so frequently. Generally, these contractures are a result of antagonistic and synergistic muscle synergies forming abnormal conditions from the trunk down to the toes. Secondary curvatures of the spine, i.e. hyperlordosis and scoliosis originate from flexion and adduction contractures of the hip. (fig. I)

The malposition of the spine renders sitting in the wheelchair for paraplegics more difficult (fig. 2) furthermore, the inclination of the pelvis may result in pressure sores above the single-loaded ischium. These initial muscle contractures, may lead in due course to articular fixation. For the therapeutic aspect we have, therefore, to distinguish between myogenic and arthrogenic contractures. They can be differentiated by examining the passive flexibility under general anaesthetic in the presence of completely relaxed muscles. To get a real degree of the motility the contractures should be mobilised for IO-I5 minutes prior to measuring. Indication and timing for surgery are determined by the measurement of the passive flexibility and X-ray. Sufficient passive motility in pure muscle contractures enables us, without difficulty, to correct the contracture by myotomies; tenotomies or neurectomies, such as myotomy of the m. iliopsoas intrapelvic, extraperitoneal resection of the obturator nerve, slicing of muscles or of the tendons of the $\mathrm{mm}$. gastrocnemii, lengthening of tendons and similar methods.

Severe (intractable) flexor spasms without arthrogenic contractures indicate treatment, for instance by anterior or posterior rhizotomy at a level of LI to SI or by partial denervation of tibial nerve branches in lower lesions, etc. Periarticular ossifications and ankylosis revealed by X-ray resulting in contractures of the joints can only be eliminated by surgical procedures on the bone. However, the type of operation-removal of the calcium deposits, resection of the head of the femor or intra and subtrochanteric osteotomy with forming a pseudoarthrosis-has to be decided individually, sometimes just during the surgical procedure.

\section{SUMMARY}

Based on the differentiation of myogenic and arthrogenic contractures, the type of the chosen procedure will prove success or failure of surgery. In principle, the malposition of a single joint must never be considered for itself, rather the entire extremity has to be regarded as a functional unit. The malposition of one joint indirectly or directly causes that of the others.

Contractures may require surgical procedures on nerves, tendons, muscles and bones. Contractures complicating spasms should be removed. The final stage of the pathological process should be waited for in cases with calcifications and ossifications.

As contractures and ankylosis are a considerable handicap to the nursing and daily activities of paraplegics, every attempt to correct the malposition is justified even if it only enables the injured to sit in an upright position. 